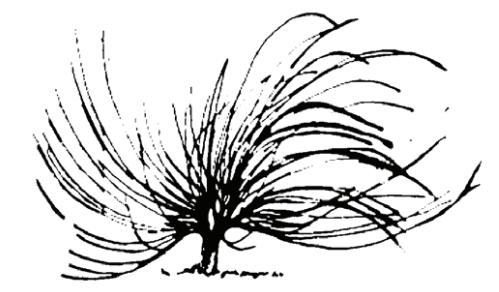

\title{
La Interacción con Angloparlantes y su Influencia sobre la Competencia Comunicativa
}

\author{
Henry Méndez Pérez ${ }^{1}$ \\ Universidad Invenio \\ Guanacaste, Costa Rica \\ henry.mendez@invenio.org
}

\begin{abstract}
Resumen
El idioma inglés se ha convertido en la lengua importante de la sociedad del conocimiento en que se desarrolla el ser humano actual. Para adquirirlo, cada día miles se matriculan e inician el sueño de poder comunicarse en esta lengua. Sin embargo, un número bastante similar abandonan las aulas, frustrados por no poder alcanzar la meta. ¿Cuáles son los factores que llevan a tantos estudiantes a tomar esta determinación? Independientemente de las razones, el aprender inglés como idioma extranjero es algo que muy pocos logran. El siguiente artículo científico tuvo como objetivo determinar los aspectos de la competencia comunicativa que mejoraron los estudiantes al interactuar semanalmente con angloparlantes. Para ello, se seleccionó un grupo de once estudiantes de la carrera de inglés como lengua extranjera de la Universidad Técnica Nacional en Costa Rica. Los resultados que se obtuvieron fueron muy interesantes ya que la pronunciación es la macro habilidad del inglés que más se les dificulta a los
\end{abstract}

Recibido: 2 de abril de 2014-Aprobado: 13 de abril de 2015

1 El maestro Henry Méndez Pérez es educador desde el año 2002. Ha trabajado en primaria, secundaria y desde hace más de 8 años en educación superior. En el año 2012, terminó una maestría virtual con el Instituto Tecnológico de Estudios Superiores de Monterrey. 
estudiantes y es justamente este aspecto el más beneficiado con las sesiones de conversación. Al final, se concluyó que la colaboración de un voluntario angloparlante en el aula es un asunto fácil, divertido, dinámico, innovador, diferente y muy sencillo de lograr.

Palabras clave: Interacción, voluntario, inglés, competencia, innovación

\begin{abstract}
English has become the language of the knowledge society in which modern human beings live. To acquire it, every day thousands enroll and begin the dream of being able to communicate using this language. However, truancy is the worst enemy and a number quite similar renounce and leave the classrooms, frustrated by the feeling of not being able to reach the goal. What are the factors that lead many students to make this decision? Whatever the reasons, learning English as a foreign language is something that very few complete. The following scientific article aimed to identify the areas of the communicative competence that students improve by weekly interacting with English speakers. To achieve the goal, a group of twelve students were selected. They were studying English as a foreign language in the Universidad Técnica Nacional in Costa Rica. The results obtained were very interesting because the aspect of the communicative competence that they find the most difficult is the one being positively affected by these conversational sessions. As a conclusion, the collaboration of an English speaking volunteer in the classroom is an easy, fun, dynamic, innovative, different and very easy issue.
\end{abstract}

Keywords: interaction, volunteer, English, competence, innovation

\title{
Introducción
}

La globalización es un fenómeno económico, político, social y educativo que ha afectado la forma de vivir de las personas y su interacción con el resto del mundo. En la era de la globalización, el mundo 
ha diluido sus fronteras generando una mayor integración e interdependencia entre muchas diferentes culturas, costumbres e idiomas. Esta situación lleva a Costa Rica a enfrentar diversos retos, uno de estos, es específicamente el desarrollo de las competencias lingüísticas. El mejorar la capacidad de comunicación multilingüe impacta positivamente la agenda de competitividad del país y su economía.

Cuando se habla de la adquisición de una segunda lengua, bien se puede decir que lamentablemente nuestras instituciones educativas han sido absorbidas por un viejo sistema de enseñanza-aprendizaje, que desde sus orígenes ha mostrado muchas debilidades y que se ha venido arrastrando desde hace mucho tiempo en nuestro querido país. Como resultado ya no se incentiva ni se premia la máxima calidad que tanto buscan las empresas en nuestros tiempos. Esto debería guiarnos a un esfuerzo colectivo e integral por parte de todos los que de una u otra forma, hacemos educación.

Mediante un esfuerzo gubernamental por proveer una mejor calidad de enseñanza, el 11 de marzo de 2008 se crea el programa Costa Rica Multilingüe según decreto $\mathrm{N}^{\circ} 34425-\mathrm{MEP}-\mathrm{COMEX}$. Dicho programa ha buscado una visible mejora hacia la educación bilingüe. Para lograrlo, una de sus primeras acciones fue dar origen a un proyecto de conversatorios culturales apoyado en los más de 33000 angloparlantes que residen en nuestro país. Este programa ha tenido como meta principal lograr un reclutamiento de voluntarios que estén dispuestos a donar una pequeña parte de su tiempo para formar grupos de conversación con personas que estén ansiosas de mejorar su nivel de inglés.

La idea es dejar a un lado el increíble bagaje de teoría que inunda los salones de clase y poner a los participantes en la verdadera práctica de lo que constituye la adquisición de una segunda lengua. Las pizarras junto con sus explicaciones gramaticales son parte del pasado con este programa y da paso a la realidad que enfrentarán todas aquellas personas que sienten el deseo de mejorar su nivel de inglés; a saber, interactuar con personas que poseen este idioma como su lengua materna y conocer los diferentes acentos que se pueden encontrar en diversas regiones del planeta.

El programa de conversatorios comunales (Ver Apéndice A) nace en Costa Rica en la provincia de Guanacaste el día martes 19 de mayo, bajo la supervisión de la recién creada Universidad Técnica Nacional, sede Guanacaste. Estos conversatorios culturales han alcanzado y dejado 
huella en cientos de personas alrededor de nuestro territorio. Amas de casa, estudiantes, niños, adultos y cualquiera que desee mejorar su nivel de inglés son bienvenidos a participar. Ver el rostro radiante de felicidad de los que son expuestos a los angloparlantes es algo indescriptible.

\section{Antecedentes}

En un mundo donde es factible saber lo que pasa en el polo opuesto al que se encuentra un individuo en un tiempo de solamente segundos, se hace cada vez más esencial el uso de una "lingua franca" (lengua franca) o "common language" -lenguaje común- (Crystal, 2003); donde todos seamos capaces de comunicarnos sin la necesidad de un intérprete. Nuestro mundo actual ha adoptado el idioma inglés como la solución a los problemas de comunicación que se han presentado con la globalización que enfrenta nuestro planeta tierra. Un estudio realizado por Graddol (2000) muestra que más de 375 millones de personas tienen inglés como lengua materna y el número de personas que lo hablan como segundo idioma ha sobrepasado a los primeros.

A pesar del debate que existe en nuestros días sobre la disputa entre el idioma inglés y el mandarín como los posibles idiomas del futuro, resulta indiscutible la influencia que el primero tiene en el nivel mundial. Culturas como las asiáticas han adoptado la idea de que un movimiento bilingüe será el que triunfe sobre ese continente (Pak, 2012). Cada día son más las personas que optan por la capacidad de comunicarse en más de un idioma y al parecer la mayoría escogen el mundialmente hablado inglés.

Este lenguaje ha llegado a ser reconocido por la inmensa cantidad de individuos que lo utilizan para comunicarse. Pero una lengua no llega a ser global por el número de sujetos que lo hablan, sino por lo que ellos representan en la sociedad (Crystal, 2003). Son los grandes políticos y gobernantes de las naciones, individuos con mucho poder, los que más se han preocupado por la adquisición de esta lengua franca.

Todo lo anterior conduce a la necesidad mundial de ser competentes en este idioma. Manuales, programas de televisión, noticias, instrucciones, todo tipo de artículos científicos y demás información relevante, se encuentra escrito en inglés. Esto ha generado que los gobiernos de cada país promuevan una enseñanza bilingüe a través de diferentes programas y proyectos. 
En esta era globalizada, el mundo ha diluido sus fronteras generando una mayor integración e interdependencia entre diferentes culturas, costumbres e idiomas. Esta situación lleva a países como Costa Rica a enfrentar diversos retos; uno de estos es específicamente, el desarrollo de las competencias lingüísticas. El mejorar la capacidad de comunicación multilingüe impacta positivamente la agenda de competitividad del país y su economía, ofreciendo asimismo ventajas a nivel de tecnología, educación, cultura, diplomacia y otras relaciones internacionales.

Para lograr una población bilingüe que sea capaz de llenar las expectativas que demanda la sociedad actual, se debe hacer un ajuste en las metodologías que se han venido utilizando desde hace mucho tiempo en las instituciones educativas. En tal sentido, las metodologías guían hacia el éxito o el fracaso. La idea de que el profesor es el que hace todo el trabajo en el aula, debe quedar en el pasado y debemos proponer, como lo plantea Cassany, Luna y Sanz (2002), un ambiente educativo donde el estudiante tenga el mayor protagonismo posible. Utilizar nuevas metodologías que tengan como eje central la innovación, deben ser parte activa en los salones de clase, especialmente, aquellos en donde se enseña una segunda lengua.

Otro aspecto que no debe dejarse de lado es la cultura propia que forma parte integral del idioma que se está aprendiendo. Es absurdo, ilógico e imposible separar un idioma de su cultura y el aprendizaje de los aspectos culturales es algo que no se puede aprender a distancia. Los sistemas educativos han fallado ampliamente en este aspecto. Cada día es más frecuente observar miles de personas que adquieren inglés como segunda lengua y no tienen la más mínima idea de todo el bagaje cultural que rodea a los países angloparlantes.

¿Cómo lograr, entonces, la inmersión de todas las personas que están aprendiendo un segundo idioma en la cultura de los países donde se hablan esas lenguas? Pocos son los que tienen la facilidad de viajar a otro país, y menos, cuando se encuentra a gran distancia de su territorio. A veces interfiere lo económico, otras veces la familia y en algunas ocasiones no es fácil gestionar una visa para entrar a ciertos países. Entonces, ¿cómo lograr un acercamiento a la cultura propia de los idiomas?

Los angloparlantes que residen en Costa Rica conocen la cultura y la viven diariamente. A partir de aquí, surge la idea en el año 2009, de utilizar este valioso recurso en la enseñanza del inglés como segunda lengua en el territorio costarricense. Se crearon grupos de conversación 
en todo el territorio, donde participan inglés hablantes junto con personas que quieren mejorar esta competencia.

Esta estrategia ha permitido, a todos los que han formado parte del programa, conocer más ampliamente la cultura angloparlante, de esta forma, estar más capacitados para enfrentar las demandas de la sociedad del conocimiento en que nos movemos. Si se dificulta moverse hasta donde está la cultura de un idioma, entonces se puede optar por traerla al ambiente donde se está aprendiendo la lengua.

\section{Marco Teórico}

En este apartado se describe, en forma general, los datos teóricos analizados en relación con la temática de la investigación. Se abordaron los elementos conceptuales, teorías e investigaciones relacionadas con la habilidad de la correcta pronunciación que desean mejorar los estudiantes de la Universidad Técnica Nacional, sede Guanacaste en Costa Rica al interactuar semanalmente con personas que poseen inglés como primera lengua.

\section{Enseñanza del inglés}

La enseñanza de un segundo idioma ha cautivado la mente de muchas personas a lo largo de los siglos. Cada día son más numerosas las instituciones que ofrecen la posibilidad de adquirir una segunda lengua y se valen de toda clase de estratagemas para atraer a los clientes. De esta forma, muchos inician un sueño que muy pocos logran finalizar. Vale la pena, ante todo, ir un poco atrás en la historia y ver la forma en que las metodologías han evolucionado este campo de la educación.

Todo se remonta a la época, cuando diversos autores comenzaron a proponer diferentes métodos de enseñanza que fueron evolucionando a lo largo de los años. Una metodología nueva desplazaba a la anterior o la enriquecía. Con el transcurso de los años, se identificaban deficiencias en los métodos y se proseguía la lucha por proponer algo que diera mejores resultados. Se trataba de una búsqueda por facilitar la adquisición de una segunda lengua (Brown, 2000). Por eso, la forma cómo se enseña un segundo idioma, ha variado ampliamente, desde la antigüedad y hasta la Edad Moderna. Pero aparte de la evolución, hay otros aspectos dignos de mencionar, que han causado un gran impacto en las instituciones educativas. 
Uno de ellos, que se considera importante abordar en este apartado, se refiere a la típica idea de que la enseñanza de segundas lenguas ha evolucionado desde un enfoque gramatical a un enfoque oral. Esto no es necesariamente cierto ya que la historia demuestra la dualidad que siempre ha existido entre estas dos corrientes. Según Soler (2002), si se remonta a 3000 años antes de Cristo, los acadios al igual que los egipcios centraban el aprendizaje de la lengua a frases completas, donde la gramática no constituía el único resorte. La tradición gramatical cobra importancia en la Edad Media y hasta la caída del Imperio Romano y la incorporación de las lenguas nacionales.

En la educación actual, donde el énfasis se le da mayormente a la producción oral, los métodos tradicionales han dado lugar a los interactivos con el objetivo de lograr alumnos más competentes en el área de la comunicación. La idea que se persigue es favorecer un aprendizaje más propio del individuo que le sirva para el área específica donde se desarrolla y que sea mayormente de producción oral (Blázquez, 2010). En Costa Rica, uno de los campos más fuertes en donde más se necesita profesional capacitado bilingüe es el turismo.

\section{Enseñanza del inglés en Costa Rica}

En Costa Rica, la adquisición de inglés como segunda lengua ha sufrido un gran cambio en los últimos tiempos. Hace algunos años, eran muy pocas las escuelas primarias donde se ofrecían a los estudiantes clases en este idioma. En la actualidad, un gran porcentaje de los estudiantes de primaria y secundaria tienen acceso a la enseñanza del inglés.

Según la Constitución Política, la educación en Costa Rica es gratuita y obligatoria desde 1980 y se puede decir, por lo tanto, que todo costarricense tiene, actualmente, derecho a la enseñanza de inglés. La adquisición de este idioma como segunda lengua en este país; sin embargo, es de reciente importancia. Córdoba, Coto y Ramírez (2005), en su estudio sobre la enseñanza de inglés en Costa Rica demuestran que la enseñanza del inglés en la escuela primaria, se da a partir del 18 de julio de 1994, a raíz de un ofrecimiento de la campaña política de José M. Figueres O. Luego, según consta en la investigación mencionada, el 8 de mayo de 1997, se declara el inglés como materia básica en primero y segundo ciclos. Un año después, en 1998, se inicia el plan piloto para incorporar el inglés en la etapa preescolar. 
Costa Rica es, no obstante, un país ampliamente alfabetizado. Datos de Coalición Costarricense de Iniciativas de Desarrollo CINDE (2011) demuestran que esta nación posee un grado de 96.9\% de alfabetización, que representa el quinto puesto en Latinoamérica. Para lograrlo, el gobierno emplea al menos el $8 \%$ del producto interno bruto a diversos programas educativos.

\section{Interacción con angloparlantes}

Al ser el idioma inglés una de las demandas fuertes de la sociedad del conocimiento, el aprendizaje de este idioma ha inducido a diversos investigadores a buscar una salida a los inconvenientes que se encuentran presentes a la hora de adquirirse. Un voluntario angloparlante puede desempeñar un rol tan importante como el del mismo facilitador en las instituciones educativas cuando se trata de la adquisición de una segunda lengua. Muchos centros de formación, afortunadamente, ya están utilizando este recurso.

Algunos autores ya se han referido a esta innovación. Singleton (1999) presenta una serie de actividades por desarrollar con voluntarios, además, menciona la inmensa ayuda que ellos pueden ofrecer en el aula. Pueden dar atención a todo el estudiantado mientras se participa en actividades grupales, o bien, podrían ofrecerles ayuda individual. La idea de Marelli $(2008$, p.7) con respecto al trabajo realizado por voluntarios en el Centro Educativo Franciscano No 1001 "San Francisco" (Santa Fe), lo expone de la siguiente forma:

El aprender el idioma por parte de los niños, se convierte en enseñar y aprender juntos -voluntario y niños- los idiomas -inglés y castellano. Ambos son educadores y aprehendientes. Igualados frente a lo que ignoran y diferenciados desde lo que saben. Ambos poseen algo valioso para intercambiar con el otro que se instala más allá del lenguaje y que se mediatiza a través de él: costumbres, comidas típicas, recreación, trabajos, ocupación del tiempo libre, moda, música, deportes y educación. En definitiva, son formas de vida lo que están intercambiando, a través del diálogo, los juegos, los almuerzos compartidos, las fotos, la bibliografía, las salidas educativas. 
El hecho de contar con un voluntario angloparlante en las clases de inglés, sea de niños, jóvenes o adultos produce justamente lo que el autor menciona en la cita anterior. Por esta razón, se está trabajando fuertemente para lograr un mayor acercamiento entre nuestra sociedad y los que tienen inglés como primera lengua.

\section{Metodología}

Este apartado muestra los aspectos fundamentales relacionados con el método de investigación utilizado en la elaboración de este estudio. Se inicia con una descripción del enfoque metodológico que se ha escogido y se justifica la selección de este. Se describen elementos tales como la muestra seleccionada y las razones por las que se procedió de esta forma. Hacia el final de la sección, se trata la manera en que se analizó la información recolectada y los instrumentos que se utilizaron para la triangulación de ella.

\section{El enfoque de la investigación}

Para la realización de este estudio científico, se seleccionó el enfoque cualitativo, que pretende proveer una explicación y una predicción de una realidad social vista desde una perspectiva objetiva $\mathrm{y}$ externa (Galeano, 2006). En este tipo de investigación, como lo afirma Barrantes (2002), el investigador es parte fundamental del proceso, con sus valores, virtudes y defectos.

\section{Participantes}

El presente artículo científico está basado en el enfoque de investigación cualitativa, por ende, se ha utilizado una muestra pequeña. En total, se seleccionaron once estudiantes, los cuales pertenecen a la generación de alumnos que se registraron en el año 2010, en la Universidad Técnica Nacional, sede Guanacaste, Costa Rica, en la carrera de inglés como Lengua Extranjera. Los participantes son jóvenes cuyas edades oscilan entre los 20 y 25 años de edad. El grupo está compuesto por ocho mujeres y cuatro varones.

El nivel de inglés que poseían los participantes, al iniciar la carrera en el año 2010, era muy similar con excepción de dos alumnos 
que habían asistido a colegios privados, por lo tanto, podían comunicarse más efectivamente en este idioma que el resto de sus compañeros. Todos han mostrado un avance significativo a un ritmo muy parejo. Actualmente, se encuentran en la etapa final para obtener el título de diplomado en inglés como lengua extranjera.

\section{Instrumentos}

Cada uno de los instrumentos busca valorar la influencia que tiene la interacción con angloparlantes en los estudiantes de inglés como segunda lengua en la Universidad Técnica Nacional, sede Guanacaste en Costa Rica. Para ello, se plantearon interrogantes que puedan reflejar el punto de vista de los sujetos de estudio. En las observaciones, se buscó identificar la veracidad de las respuestas que han provisto los estudiantes.

\section{Entrevista en profundidad}

El primer instrumento utilizado se trató de una entrevista en profundidad que fue aplicada al total de la muestra seleccionadaa, o sea, a once estudiantes. Con él se pretendía conocer más de cerca la opinión de los alumnos con respecto a las sesiones semanales de conversación (Ver Apéndice B). Con respecto a este instrumento, Callejo (2002) presenta las siguientes características: es una conversación ordinaria en donde el entrevistador y el entrevistado se turnan en la toma de la palabra con la diferencia de que el entrevistador propone temas y la persona entrevistada trata de producir respuestas aceptables. Además, el sentido pragmático se encuentra en la investigación, es un habla para ser observada, busca aproximarse a la experiencia de los sujetos y es un contrato de confesión limitada.

\section{Observación}

Se realizaron tres observaciones en total a diferentes sesiones de conversación: una al inicio del cuatrimestre, una a mediados y la última al final del período. En cada una de las observaciones, estuvieron presentes los once estudiantes y además cinco voluntarios. Para capturar la información se utilizó una guía de observación que incluía aspectos 
como tema, actividades desarrolladas, actitud de los estudiantes y de los voluntarios, entre otros. Este instrumento permitió al investigador acercarse bastante a la realidad que se vive por parte del sujeto en estudio.

\section{Diario de campo}

Este instrumento permitió al investigador anotar cada detalle relevante durante todo el proceso del estudio científico. Incluía aspectos generales como descripción, interpretación, conceptualización y observaciones generales de la información recolectada. Los objetivos perseguidos con este diario incluían la recolección de datos para luego clasificarlos, advertir las lagunas que habían quedado en la información, ver el afuera filtrado a través de la mirada del adentro y llevarse como un registro continuo y sistemático. Gutiérrez (2003) define esta herramienta como un instrumento de recolección de datos con cierto sentido íntimo que implica una descripción detallada de los acontecimientos.

\section{Resultados y Discusión}

El análisis de información ha cautivado la atención de los investigadores a través de la historia. Saber que los datos que se tienen a mano son de alto valor científico y que podrían cambiar el rumbo y destino de un grupo de personas, una comunidad, un país o inclusive del mundo entero, es fascinante. En cuanto a la investigación de tipo cualitativo, existe la paradoja de que aunque el grupo que se estudia es por lo general de pocas personas, la cantidad de información que se obtiene es bastante amplia (Álvarez-Gayou, 2005).

Para lograr conclusiones válidas y de alto valor científico, se utilizaron las siguientes fases o pasos propuestos por Álvarez-Gayou, 2005; Miles y Huberman, 1994; Rubin y Rubin, 1995: obtener la información, capturar la información, transcribir la información, ordenar los datos, codificar la información y finalmente integrar todos los datos.

Para lograr este fin se analizaron las preguntas planteadas en la entrevista que se realizó a los sujetos en estudio, es decir, a los once estudiantes de la carrera de inglés como lengua extranjera de la Universidad Técnica Nacional en Costa Rica. Luego se realizó una síntesis de la información a través de un cuadro de triple entrada en donde se resumieron los datos de cada uno de los tres instrumentos y se comparó 
cada aspecto considerado relevante. Cada opinión individual estampada en la entrevista fue considerada como relevante.

\section{Recolección y análisis de la información}

Primeramente, se realizó una observación el día 06 del mes 06 del 2012. La segunda tuvo lugar el día 23 del mes 07 y la última se realizó el día 06 del mes 08 del mismo año. Todas tuvieron lugar en un salón de clases de la Universidad Técnica Nacional en la ciudad de Liberia, Guanacaste. Como segundo instrumento, se utilizó el diario de campo en tres ocasiones en las mismas fechas que las observaciones. El último instrumento que se utilizó fue la entrevista en profundidad. Esta se aplicó a los once estudiantes el día 06 de agosto del 2012.

Cada entrevista fue realizada de manera individual y se tardó aproximadamente 30 minutos para completarla. Once entrevistas en total que serían utilizadas para analizar, comparar y dar respuesta a la pregunta de investigación. Mientras respondían a cada una de las preguntas algunos se atrevieron a ponerle un poco de humor al momento. Circularon comentarios en torno a que no habían estudiado para la entrevista, que algunos de sus compañeros estaban haciendo trampa, o bien, que cuál era el porcentaje que tenía cada pregunta.

\section{Interpretación de resultados}

Este apartado presenta la interpretación de los resultados tomando en cuenta lo que expone Flick (2007), al mencionar que la interpretación de los datos es un factor decisivo para determinar las declaraciones que pueden hacerse y las conclusiones que se pueden extraer del material empírico.

\section{Categorías}

Las categorías siguieron el mismo patrón que las preguntas. Una vez definidas, se procedió a analizar cada una de ellas individualmente.

\section{Primera categoría: razones para estudiar inglés}

En esta categoría siete de once alumnos en total respondieron que la razón era sencillamente porque les gustaba. Otras respuestas giraban 
en torno a la necesidad de poder comunicarse en un segundo idioma; mientras que para otros es meramente un reto que se han propuesto. La última contestación obtenida por parte de dos estudiantes que trabajan y que ya tienen su carrera profesional, es complementar lo que ya hacen.

\section{Segunda categoría: habilidad más difícil de desarrollar}

Conduciendo la lista de las habilidades que más difícilmente asimilan los alumnos en el proceso de enseñanza-aprendizaje de inglés como lengua extranjera, se encuentra el habla. Ocho de los once participantes consideran que esta es la mayor dificultad al momento de aprender inglés (más de una opción podía ser seleccionada). Otra habilidad considerada casi igualmente dificultosa es la escucha. Seis de los once estudiantes consideran que este es uno de los aspectos de la competencia comunicativa que más les cuesta.

\section{Tercera categoría: aspecto más complejo de la competencia comunicativa}

La pronunciación es considerada como el aspecto de la competencia comunicativa que más se les dificulta a los alumnos adquirir. Por más esfuerzo y práctica que realicen, los resultados parecen ser frustrados cuando se trata de pronunciar. Seis de once estudiantes calificaron esta habilidad como la más difícil de adquirir. Y es que acertadamente lo presentan Dziubalska y Przedlacka (2008), al mencionar que la pronunciación es más que un asunto de pragmática comunicativa; es cuestión de imagen personal. Además, los oyentes, tanto nativos como no-nativos, evalúan al hablante bajo los parámetros de su pronunciación.

\section{Cuarta categoría: experiencia en los conversatorios}

Otro aspecto que se consideró pertinente conocer es la reacción de los estudiantes en torno al beneficio que los conversatorios han tenido sobre sus vidas como aprendientes de inglés. La respuesta fue afirmativa en un 100 por ciento de los once participantes. Todos concordaron en que la experiencia ha sido muy provechosa y se refirieron a ella con términos como: interesante, enriquecedora, linda, útil, sensacional, increíble, provechosa y excelente. 


\section{Quinta categoría: alcances en el dominio del inglés}

Se han mencionado diferentes aspectos; sin embargo, el que se considera más influenciado es nuevamente la pronunciación. Seis alumnos de once en total se refirieron a este aspecto como el que más se ve afectado positivamente al interactuar con angloparlantes. Otros elementos que han sido mencionados también son la fluidez, la escucha, el acento, el ritmo, la entonación, el habla y la acentuación.

\section{Sexta categoría: dificultad para entender a los angloparlantes}

Esta categoría presenta la opinión de los estudiantes con respecto a la dificultad de los acentos. Todos concordaron en que algunos son más complicados que otros. Los estudiantes lo vivieron personalmente al interactuar con norteamericanos de diferentes regiones y también británicos. La mayoría de los alumnos se inclinaron por la opción de que las diferentes regiones, con su acento propio, es lo que hace que a veces sea tan difícil de entender. Otros agregaron que aspectos tales como la rapidez con que hablan, el ritmo, la entonación y la acentuación también afectan la comprensión.

\section{Séptima categoría: beneficios en la competencia comunicativa}

En sus respuestas a la interrogante: ¿Cómo evaluarías esta herramienta para mejorar el nivel de inglés comparada con las demás que se utilizan en el aula? Todos los alumnos respondieron positivamente. La estudiante $\mathrm{N}$ se refirió a esta metodología con las siguientes palabras: "Muy buena, es una excelente herramienta ya que se mejora aspectos como la pronunciación, la acentuación y al mismo tiempo una se va echando al agua y así se aprende bastante vocabulario." Por otra parte, la estudiante S respondió lo siguiente: "Esta herramienta es excelente ya que te ayuda a afinar el oído al escuchar los diferentes tipos de acentuación de las personas nativas de este idioma."

\section{Octava categoría: interacción y competencia comunicativa}

La pregunta planteada fue: ¿Considera usted que se mejora el nivel de inglés participando en estos conversatorios? Todos los estudiantes 
respondieron afirmativamente y no solamente señalaron sí o no, sino que agregaron comentarios al respecto. La estudiante $\mathrm{P}$ mencionó el siguiente comentario: "Sí, porque me permite evaluarme, ya que al hablar con ellos nos damos cuenta que tan bien o mal hablamos."

De esta forma, una vez analizada cada una de las categorías, ha quedado grabada la opinión de cada estudiante sobre los conversatorios con angloparlantes. Es notorio mencionar, que lo que se ha presentado en este capítulo a través de la información recolectada, es lo que el investigador creía que era la situación. Los hallazgos no han originado mayores sorpresas en cuanto a lo que la pregunta de investigación cuestionaba. Los resultados han sido satisfactorios y se presentarán detalladamente en el siguiente apartado.

\section{Conclusiones}

El idioma inglés es actualmente considerado como una herramienta fundamental para ampliar el ámbito ocupacional, favorecer la autonomía intelectual y además participar plenamente en el mundo donde la interacción entre los seres no implica necesariamente la presencia física (Ballestero y Batista, 2007). En este último apartado del artículo, se expone un resumen de los principales hallazgos obtenidos durante la investigación.

\section{Principales hallazgos}

Los descubrimientos hallados en cualquier investigación son, sin lugar a dudas, el aspecto más emocionante y de más valor para el investigador. Los que se han presentado a lo largo del desarrollo de este estudio, han guiado hacia la respuesta a la pregunta generada desde el inicio, que fue la que guió todo el proceso investigativo. La pregunta de investigación ha quedado respondida de la siguiente forma:

Primero. En la adquisición de inglés como segunda lengua, la interacción con angloparlantes desempeña un papel fundamental al que debería tener acceso cada estudiante que se encuentra en 
este proceso. El rol de un voluntario de esta categoría en el salón de clases puede ser tan fundamental como el del mismo profesor. Este puede, además, participar y guiar un sinfín de actividades, de esta forma, ayudar a que la clase sea más motivante.

Segundo. El aspecto de la competencia comunicativa que más difícilmente desarrollan los estudiantes es la pronunciación. A pesar de los diferentes cursos de fonética que forman parte del currículo de la carrera de inglés como lengua extranjera de la Universidad Técnica Nacional, los alumnos siguen enfrentando problemas con algunos de los sonidos propios del idioma meta.

Tercero. Es precisamente la pronunciación, el aspecto más beneficiado con la interacción semanal con personas que tienen inglés como primera lengua. El hecho de tener la oportunidad de escuchar detenidamente los sonidos propios del idioma inglés, de personas que lo poseen como lengua materna, permite a los estudiantes asimilarlos más eficazmente.

En respuesta a la pregunta de investigación se puede decir entonces, y en primera instancia, que la competencia comunicativa es ampliamente influenciada a través de conversatorios semanales con angloparlantes. Los estudiantes de inglés como lengua extranjera de la Universidad Técnica Nacional han demostrado un visible crecimiento en diferentes factores de la comunicación oral. Por otra parte, se ha descubierto que el aspecto más ampliamente favorecido por esa interacción es la pronunciación. Otros elementos como el ritmo, la comprensión, la fluidez, la escritura, el habla, la acentuación y la entonación se han visto igualmente favorecidos.

\section{Limitaciones}

El tiempo para realizar esta investigación se consideró la principal limitante, por el hecho de que resulta relativamente corto. Hay que avanzar en cada apartado a un ritmo que a veces parece tornarse demasiado pequeño para detallar cada aspecto como el investigador quisiera hacerlo.

La institución ofrece un apoyo limitado ya que esta debe sujetarse a una serie de reglamentos que no pueden ser violentados. La gestión de 
los permisos para hacer observaciones, entrevistas y otros instrumentos de recolección de datos a veces se tornan burocráticos, por lo tanto, se requiere de mucha paciencia y perseverancia. Para poder realizar el trabajo de campo, hubo que solicitar el visto bueno de los docentes y de los estudiantes.

Además, con respecto al campo de estudio que se está trabajando, las fuentes están en el idioma inglés, mientras que el estudio fue desarrollado en español. Esta situación afecta la rapidez con que se puede trabajar. Un último obstáculo que se presentó en el estudio científico y que se hace imposible tener control sobre este es el choque que se presenta en la comunidad educativa a la hora de que se pone en práctica una idea nueva. A pesar de que algunos aceptan con mucho entusiasmo las innovaciones, otros, tanto estudiantes como profesores siempre se opondrán al cambio.

\section{Recomendaciones}

El ámbito del proceso de aprendizaje se transforma de una manera vertiginosa. Las tradicionales instituciones educativas, sean estas presenciales o a distancia, tienen el deber de reajustar sus sistemas de distribución y comunicación (Salinas, 2004). Con el fin de lograr lo expuesto anteriormente, se presentan a continuación, dos recomendaciones prácticas derivadas de esta investigación:

- $\quad$ La educación bilingüe ha sido, es y será un campo de innovación diaria donde el educador debe buscar las mejores estrategias para hacer que su clase sea dinámica y productiva. La ayuda de un angloparlante en el salón de clases se puede convertir en una asistencia fundamental en el afán de alcanzar los mejores resultados. Es fácil, divertido, práctico, se ahorra tiempo y se le da la oportunidad a los estudiantes de enfrentar la realidad.

- $\quad$ Las universidades públicas y privadas deberían promover más este tipo de estrategias de enseñanza invirtiendo recursos y tiempo en la investigación en esta área específicamente. Además, sería de gran utilidad que existieran convenios entre entidades como EF (Education First, Educación Primero), Projects Abroad (Proyectos en el Extranjero), AFS Programas Interculturales y otras más de esta índole, con las instituciones educativas en donde se enseña inglés como lengua extranjera. 


\section{Referencias bibliográficas}

Álvarez-Gayou, J. (2005). Cómo hacer investigación cualitativa: Fundamentos y metodología. México: Paidós.

Ballestero, C. y Batista, J. (2007). Evaluación de la enseñanza del inglés con fines específicos en educación superior. Revista Omnia, 13(001), 105-129. Recuperado de http://redalyc.uaemex.mx/redalyc/pdf/737/73713106.pdf

Barrantes, R. (2002). Investigación: Un Camino Al Conocimiento. Un Enfoque Cuantitativo Y Cualitativo. EUNED. San José: Costa Rica.

Blázquez, A. (2010). Metodología de la enseñanza del inglés como segunda lengua. Innovación y experiencias educativas. Recuperado el 21 de marzo de http://www.csi-csif.es/andalucia/modules/ mod_ense/revista/pdf/Numero_30/ANTONIO_BLAZQUEZ_ ORTIGOSA_02.pdf

Brown, H. D. (2000). Teaching by Principles: an interactive approach to language pedagogy. San Francisco, California: Longman

Callejo, J. (2002). Observación, entrevista y grupo de discusión: El silencio de tres prácticas de investigación. RedALyC, 76(5), 409422. Recuperado de http://www.ecominga.uqam.ca/ECOMINGA_2011/PDF/BIBLIOGRAPHIE/GUIDE_LECTURE_2/7/7. Callejo_y_Gallego.pdf

Cassany, D., Luna, M. y Sanz, G. (2002). Enseñar Lengua (Reimpresión 8a). Barcelona, España: Graó.

CINDE, (2011). Panorama general de la educación en Costa Rica. Recuperado el domingo 29 de abril de http://www.cinde.org/attachments/079_Educacion\%20en\%20Costa\%20Rica.pdf

Córdoba, P., Coto, R. y Ramírez, M. (2005). La enseñanza del inglés en Costa Rica y la destreza auditiva en el aula desde una perspectiva histórica. Revista Electrónica Actualidades Investigativas en Educación 5(2). Recuperado de http://revista.inie.ucr.ac.cr/ uploads/tx_magazine/ingles_01.pdf

Crystal, D. (2003). English as a global language. Cambridge University Press. Recuperadoel lunes 13 de febrero2012 dehttp://www.sprachshop.com/ sixcms/media.php/811/English_as_a_grobal_lang_sample_ch.pdf

Dziubalska, K y Przedlacka J. (2008). English pronunciation models: A changing scene. Bern, Switzerland: Peter Lang AG, International Academic Publishers. 
Flick, U. (2006). Introducción a la investigación cualitativa. (2da ed.). Madrid, España: Ediciones Morata.

Galeano, M. (2004). Diseños de proyectos en la investigación cualitativa. Fondo Editorial Universidad EAFIT. Medellín: Colombia.

Graddol, D. (2000). The future of English? The English Company (UK) Ltd. Recuperado el lunes 13 de febrero 2012 de http://www.britishcouncil.org/learning-elt-future.pdf

Gutiérrez, N. (2003). Propuesta de innovación de intervención pedagógica (Tesis de Maestría). Recuperado el jueves 26 de abril de http://biblioteca.ajusco.upn.mx/pdf/20864.pdf

Marelli, L. (2008). Una experiencia intercultural de aprendizajes compartidos. UNAM. Recuperado el viernes 23 de marzo de http:// www.unam.edu.ar/2008/educacion/trabajos/Eje\%203/245\%20 -marelli.pdf

Pak, J. (2012). Is English or Mandarin the language of the future? News Magazine. Recuperado el viernes 24 de febrero 2012 de http:// www.bbc.co.uk/news/magazine-17105569

Salinas, J. (2004). Innovación docente y uso de las TIC en la enseñanza universitaria RU\&SC. Revista de Universidad y Sociedad del Conocimiento, 1(1), 1-16. Recuperado de http://redalyc.uaemex. $\mathrm{mx} /$ redalyc/pdf/780/78011256001.pdf

Singleton, K. (1999). Using Volunteers in your ESL Classroom: Suggestions for Newer Teachers. The Internet TESL Journal, 5(3). Recuperado el miércoles 21 de marzo de http://iteslj.org/Articles/ Singleton-Volunteers.html

Soler, E. (2002). Bases lingüisticas y metodológicas para la enseñanza de la lengua inglesa. Francia: Universitas. 


\section{APÉNDICES}

\section{Apéndice A: Sesión de Conversación}

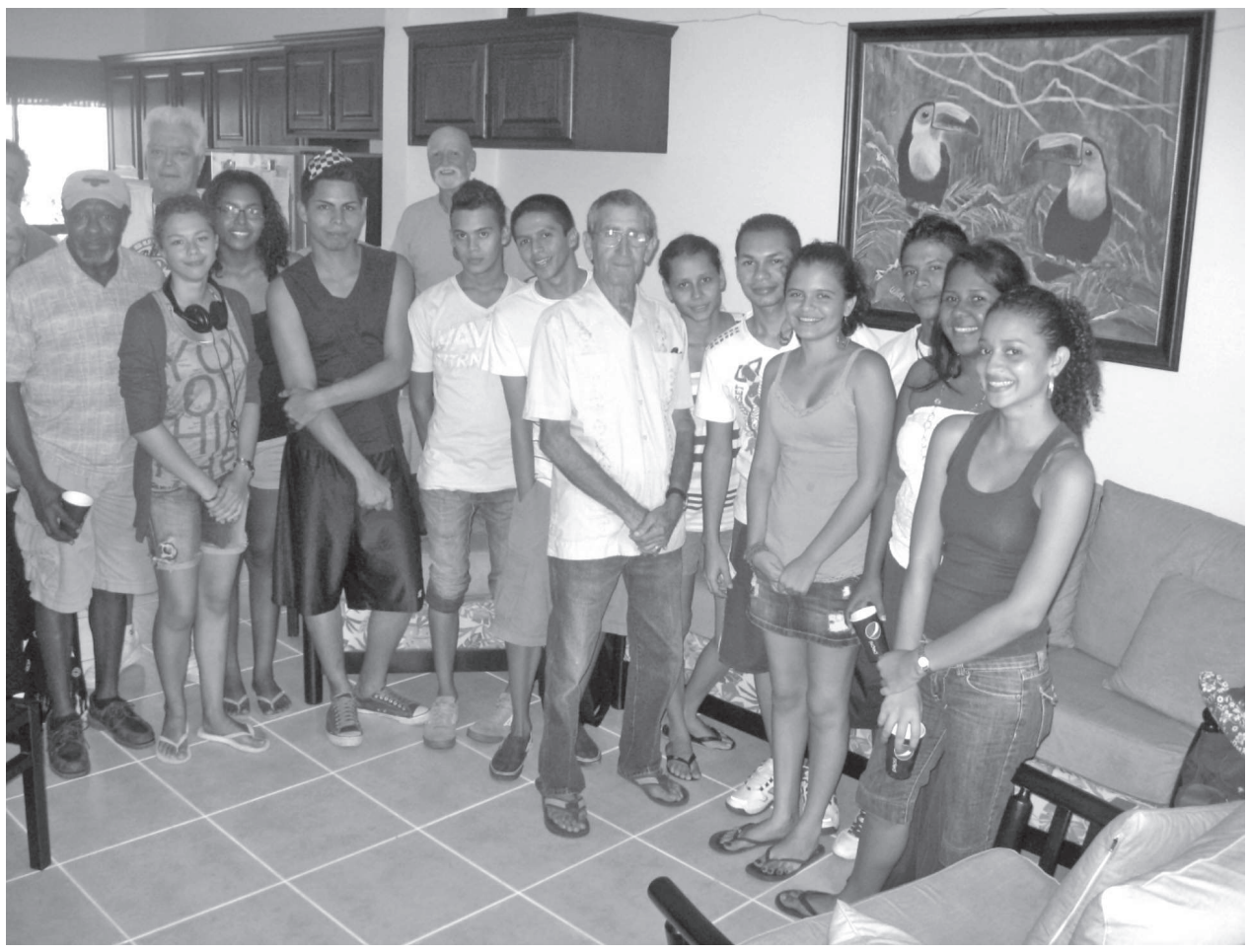

\section{Apéndice B: Entrevista en profundidad}

Entrevistas en profundidad a estudiantes de inglés de la Universidad Técnica Nacional, sede Guanacaste, Costa Rica, que participaron en los conversatorios con angloparlantes

Nombre de la Institución:

Tiempo que lleva estudiando:

Número de conversatorios:

Nombre del entrevistado:

Sexo:

Fecha: /2012 Edad: 
Estimado participante,

Mi nombre es Henry Méndez Pérez, soy estudiante de la Maestría en Educación de la Universidad Virtual del Instituto Tecnológico de Estudios Superiores de Monterrey. Les solicito su valioso apoyo para concederme una entrevista para contestar una serie de preguntas que proporcionará información valiosa para la investigación que realizo como parte del procedimiento para obtener el grado de Maestría. Agradezco de antemano su tiempo y disponibilidad.

El tema de mi estudio es la forma en que los conversatorios con angloparlantes ayudan a desarrollar los diferentes aspectos de la producción oral en los estudiantes. Las respuestas que proporcionen a las preguntas serán absolutamente confidenciales y se emplearán para la recolección y análisis de datos de este estudio.

\section{Preguntas:}

1. ¿Por qué razón decidió estudiar inglés como segunda lengua?

2. ¿Cuál es la habilidad (escucha, habla, escritura, lectura) que usted considera como la más difícil de dominar en inglés?

3. ¿Conoce usted los diferentes aspectos que conforman la producción oral del inglés? Menciónelos.

4. ¿Cuál de esos aspectos considera como el más difícil de desarrollar?

5. ¿Cómo considera la experiencia que has tenido en los conversatorios con angloparlantes?

6. Describa la experiencia, al principio y al final del período en que participó de las sesiones semanales. 
7. ¿Considera usted que ha mejorado su nivel de inglés participando en estos conversatorios?

8. ¿Cuáles aspectos de la producción oral han sido los más beneficiados?

9. ¿Hay algunos angloparlantes a los cuales es más difícil de entender que a otros? Si es así, ¿Cuáles consideras que podrían ser las razones?

10. ¿Cómo evaluarías esta herramienta para mejorar el nivel de inglés comparada con las demás que se utilizan en el aula?

La información que usted ha provisto en esta entrevista será de mucho valor para esta investigación. ¡Muchas gracias por su cooperación! 